\title{
A word from OLAW and USDA
}

In response to the issues raised in this scenario, the Office of Laboratory Animal Welfare (OLAW) and the United States Department of Agriculture, Animal and Plant Health Inspection Service, Animal Care (USDA, APHIS, AC) offer the following clarification and guidance:

This scenario raises questions regarding the IACUC chair's authority to decide whether to re-review an approved protocol, the use of designated member review (DMR) to review protocols in accordance with the Public Health Service (PHS) Policy on Humane Care and Use of Laboratory Animals (Policy) ${ }^{1}$ and the Animal Welfare Act and Regulations (AWARs) ${ }^{2}$, and conflict of interest in IACUC review.

One of the functions of the IACUC as required by the PHS Policy and the AWARs is to review concerns involving the care and use of animals at the institution ${ }^{1,2}$. Review of concerns is not a matter of choice; concerns must be brought to the IACUC's attention. It is not within the IACUC chair's authority to resolve concerns in accordance with his or her personal opinions. Concerns about animal activities at an institution must be reviewed at a convened meeting of the IACUC. In the situation described above, the IACUC should determine whether to re-review the protocol. The PHS Policy and the AWARs do not empower individual IACUC members to require the IACUC to re-review protocols. The IACUC should also verify whether institutional procedures concerning DMR were consistent with provisions of the PHS Policy and the AWARs.

Regarding DMR, if an IACUC member fails to respond to a request for DMR of a protocol on or before a predetermined deadline, OLAW and USDA allow this lack of response to indicate agreement with DMR. The PHS Policy and the AWARs require that, as a minimum, all IACUC members be given a list of proposed activities and that written descriptions be available to them ${ }^{1,2}$. Any member of the IACUC may then request review of any activity by the full committee. In the absence of such a request, the chair may appropriately designate at least one qualified person to review, approve, require modifications or request full committee review.

The PHS Policy and the AWARs clearly state that no IACUC member "may participate in the IACUC review or approval of an activity in which that member has a conflicting interest (e.g., is personally involved in the activity) except to provide information requested by the IACUC ${ }^{\prime \prime 1}$. . Conflict of interest may arise under a number of circumstances, including where "a member's personal biases may interfere with his or her impartial judgment, a member is involved in a competing research program, or access to funding or intellectual information may provide an unfair competitive advantage" 3 . Any member with a conflict of interest should inform the IACUC chair and should not participate in the IACUC review. If an investigator who has submitted a protocol or an amendment believes that an IACUC member has a potential conflict, the investigator may request that the member be excluded ${ }^{3}$. Neither recused nor excluded members may contribute to the quorum necessary to conduct IACUC business.

1. Public Health Service. Policy on Humane Care and Use of Laboratory Animals (US Department of Health and Human Services, Washington, DC, 1986; amended 2002).

2. Code of Federal Regulations, Title 9, Chapter 1, Subchapter A - Animal Welfare: Part 2 Regulations. §2.31.

3. Office of Laboratory Animal Welfare. Institutional Animal Care and Use Committee Guidebook 2nd edn. 15 (US Department of Health and Human Services, Bethesda, MD, 2002).

Patricia Brown, VMD, MS, DACLAM

Director

OLAW, OER, OD, NIH, HHS

\section{Chester Gipson, DVM}

Deputy Administrator

USDA, APHIS, AC single individual to determine the merit of complaints regarding animal use and welfare seems to be in opposition to the word and spirit of the regulations.

Wilson made errors as well. He asserted that an IACUC member has a right to request a re-review of an approved protocol if he or she believes it is necessary. The term re-review implies that a protocol may need to be approved twice. The Institutional Animal Care and Use Committee Guidebook ${ }^{1}$ clearly indicates that previously approved protocols can be discussed at convened meetings. Therefore, Wilson would have been within his rights to call for a discussion on this protocol at the next convened meeting. In our opinion, a discussion is not the same as a review.

We also noted two potential procedural errors. First, the Designated Member Review (DMR) process may need to be reviewed and changed to prevent this type of complaint. One weakness in the DMR process at many institutions is that the committee accepts a lack of reply to a request for DMR as an approval to go ahead with the DMR. This could be avoided by requiring each IACUC member to respond to each DMR request before proceeding with the DMR. At a minimum, each member should acknowledge receipt of the request. The Public Health Service provides guidance for DMR in its Notice NOT-OD-09-035 (ref. 2). Although this Notice does not require that each IACUC member respond to DMR requests, requiring members to reply to each DMR request indicating whether or not they request full committee review for the protocol will confirm that each member had the opportunity to request full committee review.

The second procedural error involves a conflict of interest (COI). The Institutional Animal Care and Use Committee Guidebook ${ }^{1}$ provides examples of COI, one of which states that an IACUC member's personal biases may interfere with his or her impartial judgment. If this is the case, then the investigator submitting the protocol (in this scenario, Ross) can request that the biased member (in this scenario, Wilson) be excluded from the review of his or her protocol. It seems reasonable that if the IACUC chairman has knowledge of a conflict between an IACUC member and investigator, he could excuse that IACUC member from review of that investigator's protocol on the basis of the COI without a request from the investigator.

1. Office of Laboratory Animal Welfare. Institutional Animal Care and Use Committee Guidebook (US Department of Health and Human Services, Washington, DC, 2002; reprinted 2008).

2. Office of Laboratory Animal Welfare. Guidance to IACUCS Regarding Use of Designated Member Review (DMR) for Animal Study Proposal Review Subsequent to Full Committee Review (FCR). Notice NOT-0D-09-035. (National Institutes of Health, Washington, DC, 8 January 2009).

Bell is Consultant, Bio-Medical Research Resources, Manchester, MD, and Scorpio is Clinical Veterinarian at the Department of Comparative Medicine, Johns Hopkins Medical Institutions, School of Medicine, Baltimore, $M D$. 